\title{
Announcement
}

\section{Magnetic Resonance Techniques in Multiple Sclerosis}

5th Advanced Course

'From Inflammation to Neurodegeneration' and

'Primary Progressive Multiple Sclerosis'

San Raffaele Hospital, Milan, Italy, May 25-26, 2001

For further information, please contact:

EEM European Congress Services

Via Tiburtina 646

I-00159 Rome (Italy)

Tel. +39064382599

Fax +39064382309

E-Mail eem@eemservices.com 